\title{
The Ventral Striato-Pallidal Pathway Mediates the Effect of Predictive Learning on Choice between Goal-Directed Actions
}

\author{
Beatrice K. Leung and Bernard W. Balleine \\ Brain and Mind Research Institute, University of Sydney, Camperdown, NSW 2050, Australia
}

The nucleus accumbens shell (NAc-S) plays an important role in the way stimuli that predict reward affect the performance of, and choice between, goal-directed actions in tests of outcome-specific Pavlovian-instrumental transfer (PIT). The neural processes involved in PIT downstream of the ventral striatum are, however, unknown. The NAc-S projects prominently to the ventral pallidum (VP), and in the current experiments, we assessed the involvement of the NAc-S to VP projection in specific PIT in rats. We first compared expression of the immediate-early gene c-Fos in the medial (VP-m) and lateral (VP-l) regions of the VP and in addition, used the retrograde tracer Fluoro-gold combined with c-Fos to assess the involvement of these pathways during PIT. Although there was no evidence of differential activation in neurons in the VP-l, the VP-m showed a selective increase in activity in rats tested for PIT compared with appropriate controls, as did NAc-S neurons projecting to the VP-m. To confirm that VP-m activity is important for PIT, we inactivated this region before test and found this inactivation blocked the influence of predictive learning on choice. Finally, to confirm the functional importance of the NAc-S to VP-m pathway we used a disconnection procedure, using asymmetrical inactivation of the NAc-S and either the ipsilateral or contralateral VP-m. Specific PIT was blocked but only by inactivation of the NAc-S and VP-m in contralateral hemispheres. These results suggest that the NAc-S and VP-m form part of a circuit mediating the effects of predictive learning on choice.

\section{Introduction}

The ability of animals to extract predictive information from the environment to inform future actions is critical for normal decision-making. This capacity is studied in the laboratory using the outcome-specific Pavlovian-instrumental transfer (PIT) paradigm in which a stimulus predicting a specific outcome typically biases choice toward actions earning that predicted outcome. It is clear, therefore, that PIT is an integrative process; instrumental learning allows animals to encode the consequences of their actions, and through integration with Pavlovian predictive information, to evaluate the relative values of those consequences to choose between competing courses of action. At a neural level many of the structures that have been implicated in PIT also influence the instrumental response-outcome or Pavlovian stimulus-outcome associations (Corbit and Balleine, 2005; Corbit and Janak, 2007; Ostlund and Balleine, 2007, 2008). Only the nucleus accumbens shell (NAc-S) has been found to play a critical role in the integration of the associative processes involved in PIT (Corbit et al., 2001; Corbit and Balleine, 2011; Laurent et al., 2012). Beyond the NAc-S, however, it is not known how this

Received April 22, 2013; revised June 21, 2013; accepted July 20, 2013.

Author contributions: B.K.L. and B.W.B. designed research; B.K.L. performed research; B.K.L. analyzed data; B.K.L. and B.W.B. wrote the paper.

This research was supported by funding from the National Health and Medical Research Council, Grant No. 633267, and an Australian Laureate Fellowship from the Australian Research Council to B.W.B.

Correspondence should be addressed to Dr Bernard W. Balleine, Brain and Mind Research Institute, University of Sydney, 100 Mallett Street, Camperdown, NSW 2050, Australia. E-mail: bernard.balleine@sydney.edu.au.

DOI:10.1523/JNEUROSCI.1697-13.2013

Copyright $\odot 2013$ the authors $\quad 0270-6474 / 13 / 3313848-13 \$ 15.00 / 0$ effect is mediated; although PIT presumably involves NAc-S efferents on the ventral basal ganglia, which pathways are critical remains uninvestigated.

As a major target of the NAc-S, the ventral pallidum (VP) has a strong claim to involvement in PIT. The VP is widely recognized as playing an important role in multiple incentive motivational processes as well as in motor output (Austin and Kalivas, 1990; Smith and Berridge, 2005; Smith and Tindell, 2009). As with the accumbens, however, the VP is a heterogeneous structure that can be anatomically and neurochemically divided into distinct rostromedial (VP-m) and caudolateral (VP-l) subregions. In addition, these regions also differ considerably in their afferent and efferent connections; whereas the nucleus accumbens core (NAc-C) preferentially projects to the VP-l, which then projects to the subthalamic nucleus and substantia nigra, the Nac-S predominately projects to the VP-m which projects to both the ventral tegmental area and mediodorsal thalamus (Zahm and Heimer, 1990; Zahm et al., 1996; Zhou et al., 2003; Tripathi et al., 2010). Nevertheless, these projections are not completely segregated and results from both anatomical and behavioral studies of these ventral striato-pallidal pathways suggest that either could potentially facilitate the effects of Pavlovian cues on instrumental performance (Kalivas et al., 1999; Zahm, 2000; Corbit and Balleine, 2011).

The current study aimed first to investigate whether the VP-m or VP-l is the more important for PIT. To do so we used c-Fos immunohistochemistry combined with behavioral treatments, comparing the effects of PIT with specific controls, to establish whether neurons in the VP-m or VP-l were involved in PIT. We 
found evidence for involvement of VP-m neurons and, using the retrograde tracer Fluoro-gold (FG) combined with c-Fos, for the NAc-S to VP-m pathway in PIT. In subsequent experiments we investigated the necessity of this pathway for PIT using pharmacological inactivation of the VP-m and disconnection of the NAc-S from the VP-m using asymmetrical inactivation.

\section{Materials and Methods}

\section{Overview of experiments}

Three experiments were conducted to assess the role of the VP-m in cue-guided action selection using a specific PIT paradigm. Experiment 1 used the activity marker c-Fos to investigate the activity of neurons in VP-m and the VP-l in animals that were given sessions of Pavlovian and then instrumental training followed by either: (1) a specific PIT test, (2) exposure to the Pavlovian predictors only without the opportunity to lever press, or (3) the opportunity to lever press only without exposure to the Pavlovian conditioned stimuli. Given the dominance of projections from the NAc-S to the VP-m we also wished to assess the involvement of the NAc-S to VP-m pathway in PIT and so gave rats an infusion of the retrograde tracer FG unilaterally into the VP-m with the aim of assessing activation of NAc-S neurons projecting to this region in each of the above groups based on FG and c-Fos double-labeling. Experiment 2 assessed the effects of local pharmacological inactivation of the VP-m during PIT using local infusion of the GABA-A agonist muscimol. Animals received an infusion of muscimol before test. Experiment 3 assessed whether the NAc-S to VP-m pathway is important during specific PIT by disconnecting these structures using asymmetrical infusions of muscimol into the NAc-S and either the ipsilateral or contralateral VP-m (the control group and disconnection group, respectively).

\section{Experiment 1: assessment of the role of NAc-S projections to the VP-m and VP-l in PIT \\ Subjects}

Forty experimentally naive male Long-Evans rats obtained from the Laboratory Animal Services (University of Sydney, Australia) were used as subjects. Rats were housed in a climate-controlled colony room and were maintained on a $12 \mathrm{~h}$ light/dark cycle. Rats were housed in twos or threes and handled daily for $5 \mathrm{~d}$ before surgery. During training and testing, rats were kept on a food deprivation schedule to maintain them at $\sim 85 \%$ of their free-feeding weight. The Animal Ethics Committee at the University of Sydney approved all experimental procedures.

\section{Surgery}

Stereotaxic surgery was conducted under isoflurane anesthesia (5\% induction; $1-2 \%$ maintenance). Animals were placed in a stereotaxic frame (Stoelting) and received a subcutaneous injection of $0.1 \mathrm{ml}$ of Rymadil and also a $0.1 \mathrm{ml}$ injection of bupivicane hydrochloride at the incision site. An incision was made to expose the scalp and the incisor bar was adjusted to align bregma and lambda on the same horizontal plane. Small holes were drilled above the targeted coordinates of the VP-m [anteroposterior $(\mathrm{AP}):+0.4 \mathrm{~mm}$, mediolateral $(\mathrm{ML}):+1.6 \mathrm{~mm}$ relative to bregma, dorsoventral (DV): $-8.2 \mathrm{~mm}$ relative to skull; Paxinos and Watson, 1998] and $64.4 \mathrm{nl}$ of a 3\% FG (Fluorochrome) solution diluted in $0.9 \%$ saline was injected unilaterally into the VP-m. The injector was left in place for $5 \mathrm{~min}$ to allow for diffusion before being retracted. After surgery, all rats were given a $0.4 \mathrm{ml}$ intraperitoneal injection of procaine penicillin solution $(300 \mathrm{mg} / \mathrm{kg})$ and $3 \mathrm{~d}$ to recover.

\section{Apparatus}

Training and testing took place in 16 Med Associates operant chambers enclosed in sound- and light-attenuating shells. Each operant chamber was equipped with a pellet dispenser and a pump fitted with a syringe that when activated, delivered a $45 \mathrm{mg}$ grain pellet (Bio-serve) or $0.1 \mathrm{ml}$ of a $20 \%$ sucrose solution respectively, into a recessed food magazine inside the chamber. An infrared beam crossed the magazine to detect head entries. The chambers were fitted with two retractable levers that could be inserted to the left and the right of the magazine. Each chamber also had a $3 \mathrm{~W}, 24 \mathrm{~V}$ house light that provided illumination inside the enclosed chamber, a white noise generator, and a $28 \mathrm{~V}$ DC mechanical relay that delivered a $2 \mathrm{~Hz}$ clicker stimulus. Two microcomputers running Med Associates propriety software controlled all experimental events and recorded responses.

\section{Behavioral procedures}

Pavlovian training. All rats were given eight daily sessions of Pavlovian training. Each session was $60 \mathrm{~min}$ in duration. During the session, rats received 2 min presentations of two conditioned stimuli (CS; white noise or clicker), which were paired with the delivery of a different outcome (sucrose or pellets) on a random time $30 \mathrm{~s}$ schedule throughout the CS. Each CS was presented four times in a pseudorandom order with a variable intertrial interval (ITI) that averaged to $5 \mathrm{~min}$. Half of the rats received the white noise paired with pellets and clicker paired with sucrose solution, and the other half received white noise paired with sucrose solution and clicker paired with pellets. Magazine entries throughout the session were recorded and separated into a CS period and an interval before CS presentations of equal length (Pre-CS; 2 min).

Instrumental training. After Pavlovian training, rats received $10 \mathrm{~d}$ of instrumental training. Rats were trained in separate sessions to perform left and right lever press responses for different outcomes (sucrose or pellets). Outcomes were counterbalanced with respect to Pavlovian training such that half of each CS-outcome group received pellets for left lever press responses and sucrose for right lever press responses and the other half of each group had the opposite pairings. Each session ended when 20 outcomes had been earned or after $30 \mathrm{~min}$ had elapsed. For the first $2 \mathrm{~d}$, lever presses were continuously reinforced (CRF), such that each lever press earned an outcome. Subsequently, the probability of reinforcement for responses shifted to a random ratio (RR) schedule. A RR5 $(p=0.2)$ schedule was used on days $3-5$ and a RR10 $(p=0.1)$ schedule was used on days $6-10$. The rate of responding on the lever was recorded for each session and averaged across the two sessions each day.

Pavlovian-instrumental transfer. After training, animals were assigned to one of three test conditions counterbalanced with respect to their performance during Pavlovian and instrumental training. One group of animals received a specific PIT test (Group PIT; $n=14$ ). Throughout the test session, both levers remained inserted into the chamber and no outcomes were delivered. Responding on both levers was first extinguished for $8 \mathrm{~min}$ to reduce baseline performance. Subsequently, each CS was presented four times over the next $40 \mathrm{~min}$ in the following order: clicker-noise-noise-clicker-noise-clicker-clicker-noise. Each CS lasted 2 min and had a fixed ITI of $3 \mathrm{~min}$. Two control groups were compared with the PIT group to assess the level of c-Fos expression that is specific to PIT. In one control condition, there were no presentations of the CSs but both levers remained inserted into the chamber and there were no outcomes delivered (Group LP; $n=13$ ). In another control condition, animals received the same order of presentations of the CSs at the same time as animals in Group PIT test but both levers were retracted throughout the session and no outcomes were delivered (Group CS; $n=13$ ). All test conditions were the same in duration and animals in Group LP were tested separately from Groups PIT and CS to avoid any influences of the CSs. Magazine entries and lever pressing rates were recorded throughout the session and responses in Groups PIT and CS were further separated into Pre-CS and CS periods.

\section{Histology}

All animals were perfused transcardially with a $4 \%$ paraformaldehyde PBS (0.1 M, pH7.2) solution (PFA) at $2 \mathrm{~h}$ from the start of test. Brains were extracted and kept in PFA solution overnight before being transferred to a $30 \%$ sucrose PBS solution. Brains were frozen and $30 \mu \mathrm{m}$ sections were then collected on a freezing microtome and kept at $-20^{\circ} \mathrm{C}$ in a cryoprotective solution. Sections containing the VP between +0.7 $\mathrm{mm}$ to -0.4 relative to bregma (Paxinos and Watson, 1998) were selected and mounted on slides to verify that FG injections were within the boundaries of the targeted area. Animals that had injections outside the region were removed from any FG analyses. In addition, separate sections of the VP were processed for parvalbumin immunoreactivity. These sections were used to delineate the boundaries of the VP from its sur- 
rounding structures, particularly the extended amygdala regions surrounding the VP (Zahm et al., 2003) for the volumetric analysis of c-Fos labeling.

\section{Immunohistochemistry}

Sections of the VP and NAc-S were processed for either parvalbumin immunoreactivity or c-Fos immunoreactivity, the latter to assess neuronal activity in each of these regions for each test condition. Briefly, sections were given three $10 \mathrm{~min}$ rinses in PBS, then transferred to a solution of $0.5 \%$ Triton X-100 and $10 \%$ normal goat serum in PBS for $2 \mathrm{~h}$. Sections were then incubated in a polyclonal rabbit anti-c-Fos antibody (1:500; sc-52, Santa Cruz Biotechnology) or a mouse anti-parvalbumin antibody (1:1000; MAB1572, Millipore) with $0.2 \%$ Triton X-100 and 2\% normal goat serum in $\mathrm{PBS}$ for $48 \mathrm{~h}$ at $4^{\circ} \mathrm{C}$. Sections were given three 10 min PBS rinses before they were incubated in an AlexaFluor 546conjugated goat anti-rabbit secondary antibody or an AlexaFluor 647conjugated goat anti-mouse antibody (1:1000; Invitrogen) for $90 \mathrm{~min}$. Sections were given three more 10 min PBS rinses before they were mounted on slides and coverslipped with Fluoromount-G (Southern Biotech).

\section{Fluorescence imaging and quantification of immunoreactive cells}

Injection sites and the rostral-caudal spread of FG injections were observed with an Olympus Fluoview FV10i microscope, and compared with the rat brain atlas of Paxinos and Watson (1998). Higher resolution confocal images of the VP were taken at $10 \times$ magnification in the contralateral side of the injection for quantification of c-Fos immunoreactivity. Sections selected for each region were according to the rat brain atlas of Paxinos and Watson (1998). Rostral VP-m sections were selected between +0.48 to +0.20 from bregma. Sections considered VP-l were between -0.30 to -0.60 from bregma. Based on VP sections processed for parvalbumin, a region of interest (ROI) was outlined and measured using ImageJ (http://rsb.info.nih.gov/ij) at different coronal planes and three sections per animal were quantified in each region using the standard ROIs. The number of c-Fos-positive neurons for each section was counted and expressed as square millimeters using the area of the ROIs.

For the analysis of NAc-S, sections selected were between +1.60 and +1.80 from bregma. Confocal images were acquired at $20 \times$ magnification using a Zeiss LSM 710 confocal microscope equipped with 405 and $561 \mathrm{~nm}$ diode lasers for FG-labeled cells and c-Fos immunoreactivity. Images taken remained within the boundaries of NAc-S and were assessed using ImageJ. Neurons expressing c-Fos alone, FG alone, and c-Fos double-labeled with FG were quantified in three sections per animal.

\section{Experiment 2: the effect of inactivation of the VP-m on PIT Subjects and apparatus}

Sixteen experimentally naive male Long-Evans rats obtained from the Laboratory Animal Services (University of Sydney, Australia) were used as subjects. The housing and apparatus used were identical to those in Experiment 1. Animals were placed on food deprivation and handled daily for five $\mathrm{d}$ before training.

\section{Surgery}

Stereotaxic surgery was conducted under isoflurane anesthesia using the procedures described by Experiment 1. Twenty-six gauge guide cannulae (Plastics One) were implanted bilaterally in the VP-m. To accommodate both guide cannulae, two sets of coordinates were used (relative to bregma and skull): on the left: AP: $+0.4 \mathrm{~mm}$, ML: $+1.6 \mathrm{~mm}$, DV: -7.2 $\mathrm{mm}$; and on the right: AP: $+0.4 \mathrm{~mm}$, ML: $-3.1 \mathrm{~mm}, \mathrm{DV}:-7.3 \mathrm{~mm}$ at a $10^{\circ}$ angle toward the midline. The DV coordinates of the guide cannulae were made $1 \mathrm{~mm}$ above the intended infusion site (DV: $-8.2 \mathrm{~mm}$ on the left, $-8.3 \mathrm{~mm}$ on the right) and were held in place with screws and dental cement. A dummy cannula was kept in the guide at all times except during the microinfusions.

\section{Drug and infusions}

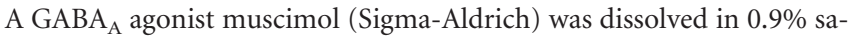
line to the final concentrations of either $0.1 \mathrm{mg} / \mathrm{ml}$ or $0.5 \mathrm{mg} / \mathrm{ml}$. Animals received either an infusion of the drug muscimol or the vehicle saline 20 min before each test. Half of the animals received the drug infusion on the first day and the vehicle infusion on the second day, whereas the other half received the vehicle infusion on the first day and the drug infusion on the second day. The order of infusions was counterbalanced with training.

At the time of infusion, dummy cannulae were removed and the infusion cannulae were lowered into the guide extending $1 \mathrm{~mm}$ below the guide tip. Infusion cannulae were connected to $25 \mu \mathrm{l}$ Hamilton syringes fitted on an infusion pump that delivered $0.2 \mu \mathrm{l}$ of the drug or vehicle at a rate of $0.1 \mu \mathrm{l} / \mathrm{min}$ to each hemisphere. The infusion cannulae were left in place for an additional $2 \mathrm{~min}$ before being removed and replaced by the dummy cannulae. Rats returned to their home cages for $15 \mathrm{~min}$ before testing. No gross motor deficits were observed in any animal after infusion of either dose.

\section{Behavioral procedures}

Pavlovian and instrumental training. Rats received Pavlovian conditioning according to procedures described in Experiment 1. Instrumental training procedures were also similar to those mentioned above. Animals first received $7 \mathrm{~d}$ instrumental training ( $2 \mathrm{~d}$ on CRF, $3 \mathrm{~d}$ on RR 5 schedule, and $2 \mathrm{~d}$ on RR10 schedule). After this initial training, the rats were given free access to food for $2 \mathrm{~d}$ before undergoing surgery to implant the guide cannulae. This was done to minimize the length of time rats had guide cannulae implanted. After $3 \mathrm{~d}$ of recovery, rats were placed back on food deprivation. Rats then received three additional days of instrumental training on a RR10 schedule.

Pavlovian-instrumental transfer. Animals were given two specific PIT tests, one under the high and then a second under the low concentration of the drug as described for Experiment 1 . The test order was not counterbalanced. For each dose, animals received two tests on consecutive days, one on each day. After testing under the high concentration, animals were given $2 \mathrm{~d}$ of instrumental retraining to return their lever press rate to that on the last day of instrumental training before being tested using the same procedure for the lower concentration of drug.

\section{Histology}

At the end of the experiment, animals received a lethal dose of sodium pentobarbital. Brains were extracted and $40 \mu \mathrm{m}$ coronal sections of the VP were collected on slides. Sections were then stained with cresyl violet to verify the location of cannulae tips. Placements that were outside the boundaries of the targeted region or with excessive damage from infusions were excluded from further analyses.

\section{Experiment 3: effect of disconnection of the NAc-S and VP-m on PIT}

\section{Subjects and apparatus}

Twenty-six experimentally naive male Long-Evans rats obtained from the Laboratory Animal Services (University of Sydney, Australia) were used as subjects. The housing and apparatus used were identical to those described above. Animals were placed on food deprivation and handled daily for $5 \mathrm{~d}$ before training.

\section{Surgery}

Stereotaxic surgery was conducted under isoflurane anesthesia using methods described above. Rats in the contralateral group $(n=13)$ had guide cannulae implanted in the NAc-S and VP-m in opposite hemispheres whereas rats in the ipsilateral control group $(n=13)$ had both guide cannulae implanted in the same hemisphere, leaving the other hemisphere intact. Similar to Experiment 2, guide cannulae were implanted at different angles due to the proximity of the targeted regions. The targeted coordinates for NAc-S were as follows: AP: $+1.7 \mathrm{~mm}$, ML: $\pm 3.6 \mathrm{~mm}, \mathrm{DV}:-7.1 \mathrm{~mm}$ at a $20^{\circ}$ angle toward the midline, and the targeted coordinates for the contralateral or ipsilateral VP-m were as follows: AP: $+0.4 \mathrm{~mm}, \mathrm{ML}: \pm 0.8 \mathrm{~mm},-7.1 \mathrm{~mm}$ at a $5^{\circ}$ angle away from 
A

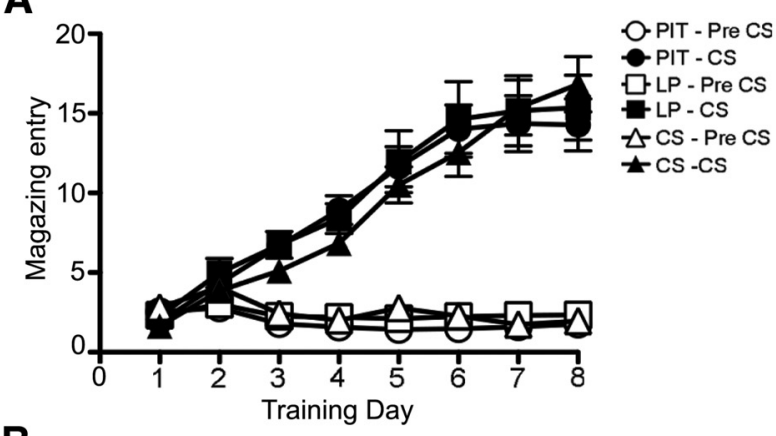

B

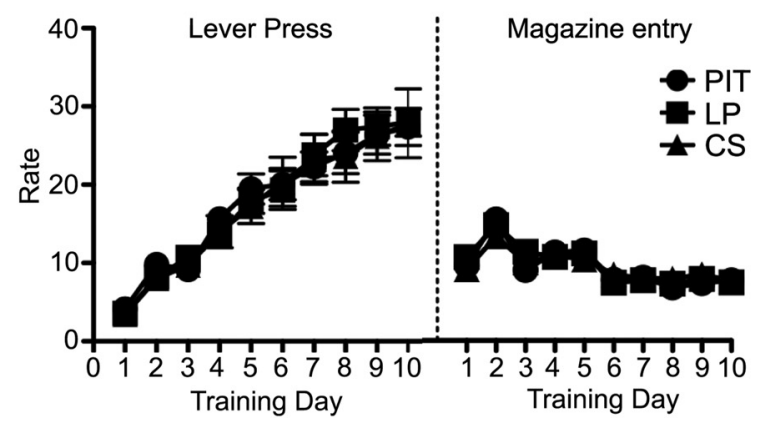

C

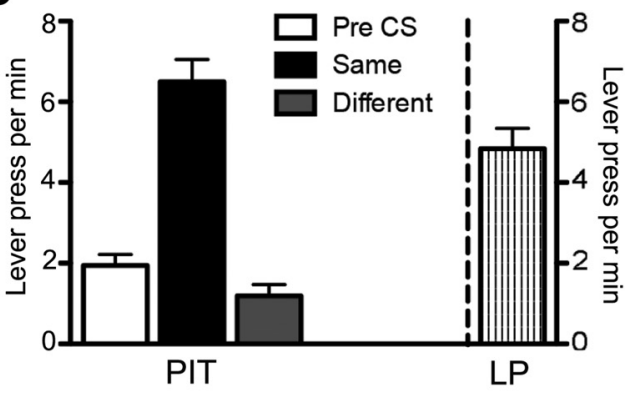

D

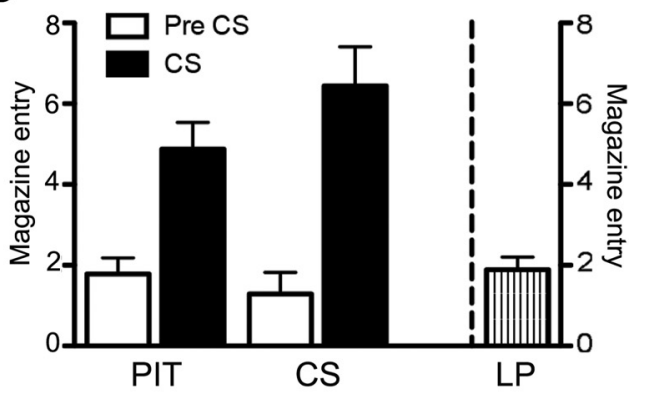

Figure 1. Experiment 1. Performance during Pavlovian and instrumental training. A, Pavlovian conditioning as the rate of magazine entries per minute during the $C S$ and Pre-CS periods over sessions in Groups PIT, LP, and CS. B, Acquisition of instrumental conditioning showing performance on lever pressing and magazine entries per minute over sessions in Groups PIT, LP, and CS. C, Lever pressing performance of Group PIT and LP during test condition. Left, Performance of Group PIT is shown as lever pressing per minute during the Pre-CS period (Pre-CS), and separately during the CS period, for responding on the lever that, in training, delivered the same outcome as that predicted by the CS (Same) and on the lever that had delivered the different outcome (Different). Right, Performance of group LP is shown as lever presses per minute throughout the entire session. $D$, Magazine entries per minute of Groups PIT, LP, and CS during the test. Left, Magazine entries for group PIT and CS are separated into Pre-CS and CS periods. Right, Magazine entries for group LP throughout the entire session. Error bars indicate SEM.

the midline. The DV coordinates of guide cannulae were made $1 \mathrm{~mm}$ above the targeted infusion site (NAc-S: $-8.2, \mathrm{VP}-\mathrm{m}:-8.2)$. The left and right position of the cannulae was counterbalanced within each group.

\section{Drug and infusion}

Due to the close proximity of the two structures, a form of muscimol with a fluorescent tag (M-23400, Invitrogen) was used to identify any diffusion of the drug into its neighboring region. The fluorescent muscimol (fMUS) was dissolved in a $40 \%$ dimethyl sulfoxide (DMSO) PBS solution to a final concentration of $0.5 \mathrm{mg} / \mathrm{ml}$. A total volume of $0.2 \mu \mathrm{l}$ to each region was infused at a rate of $0.1 \mu \mathrm{l} / \mathrm{min}$ and left for a further $2 \mathrm{~min}$ after infusion before the infusion cannulae were removed. Rats received either the drug fMUS or the vehicle $40 \%$ DMSO PBS on each test. The order of drug or vehicle infusion was counterbalanced and infusions and test sessions were identical to those used in Experiment 2.

\section{Behavioral procedures}

Rats received identical Pavlovian and instrumental training to that described in Experiment 2. Again surgery was given during an interruption in instrumental training. After training, animals were given two specific PIT tests over two consecutive days, one on each day, following infusions of either drug or vehicle. The order of the tests (drug and vehicle) was counterbalanced with half the animals tested under vehicle then drug, whereas the remainders were tested under drug then vehicle.

\section{Histology}

Eight rats were perfused transcardially with $4 \%$ PFA immediately after the final test. Cannulae were removed and brains were extracted and postfixed overnight, after which $30 \mu \mathrm{m}$ sections were collected to verify the spread of the drug. All other animals were given a lethal dose of sodium pentobarbital. Brains were extracted and $40 \mu \mathrm{m}$ coronal sections were collected on slides. Sections were then stained with cresyl violet to verify the location of cannulae tips. Placements that were outside the boundaries of the targeted region or with excessive damage from infusions were excluded from further analyses.

\section{Statistical methods}

Behavioral procedures. All analyses were conducted using mixed-model ANOVA followed by simple effects analyses to establish the source of any significant interactions.

Neuronal quantifications. Analyses of neuronal quantifications in each region were conducted using between-subjects ANOVAs. Correlations of c-Fos expression with behavioral performances were conducted using Pearson's $r$ test. Correlations in each region were calculated by comparing c-Fos expression with (1) the net PIT performance (performance on lever associated with the same outcome as the CS minus baseline from performance on the lever associated with the different outcome minus baseline) in Group PIT, (2) the overall lever pressing rates with Group PIT and Group LP combined, and (3) the elevation ratio of magazine entries during the CS from Pre-CS with Group PIT and Group CS.

\section{Results}

Experiment 1: assessment of the role of NAc-S projections to the VP-m and VP-l in PIT

Pavlovian and instrumental training

The FG infusion did not affect animals' ability to acquire both Pavlovian and instrumental conditioning, shown in Figure $1 A, B$, respectively. All three groups had similar rates of conditioned magazine entries during Pavlovian conditioning and lever pressing rates during instrumental training. During Pavlovian conditioning the difference between head entries during the Pre-CS and CS periods increased over sessions. A mixed ANOVA conducted using factors of Group (PIT, LP, and CS), CS period (Pre-CS and CS), and Session found an effect of CS period $\left(F_{(1,37)}=202.05, p<0.001\right)$, of Session $\left(F_{(7,259)}=63.86, p<\right.$ 
$0.001)$ and a CS period by Session interaction $\left(F_{(7,259)}=92.16\right.$, $p<0.001)$. There was no significant effect of Group or any interactions involving Group as a factor (largest value of $F_{(14,259)}=$ $1.02, p=0.44)$. During the instrumental training phase, rates of responding on the levers increased over days as the ratio requirement increased. A mixed ANOVA conducted using factors of Group and Session found an effect of Session $\left(F_{(9,333)}=106.53\right.$, $p<0.001)$ but neither an effect of Group nor an interaction between these factors (all values $F<1$ ). Magazine entries during instrumental training decreased as the ratio requirement increased over sessions. A mixed ANOVA conducted on magazine entries using factors of Group and Session found an effect of Session $\left(F_{(9,333)}=42.29, p<0.001\right)$ but neither an effect of Group nor an interaction between these factors (largest value $\left.F_{(18,333)}=1.37, p=0.14\right)$.

\section{Pavlovian-instrumental transfer test}

Only rats in the PIT group received the full PIT test; the control groups receiving either the levers but no presentation of the CSs (Group LP) or the CSs but no presentation of the levers (Group $\mathrm{CS}$ ). Comparing the lever press performance between group PIT and LP, the rate of lever pressing averaged across levers over the course of the 40 min test after the extinction period was $5.89 \pm$ 0.25 lever presses per minutes for group PIT and $4.83 \pm 0.51$ lever presses per minutes for group LP (Fig. $1 C$, right). ANOVA with factors of Group (PIT and LP) and Performance revealed no significant difference in the overall rate of lever pressing between the two groups $\left(F_{(1,25)}=3.66, p=0.06\right)$. However, because group PIT also received presentations of the CS, their performance was further analyzed with the CS period as a factor.

Figure $1 C$ shows the results presented as the rate of responding (lever press per min) at test for group PIT, averaged across levers, during a period when no CS was presented (Pre-CS), during the CS with which it shares the same outcome as a particular lever (Same) and during the CS with which it shares a different outcome with a particular lever (Different). It is clear from the figure that animals in group PIT showed a robust specific PIT effect; animals increased performance on the lever that shared the same outcome as predicted by the CS. ANOVA conducted using a factor of CS identity (Pre-CS, Same and Different) found an effect of CS identity $\left(F_{(2,26)}=38.29, p<0.001\right)$. Simple effect analyses conducted on each level of CS identity found no differences in rates of responding between Pre-CS and Different $\left(F_{(1,13)}=0.29, p>0.05\right)$ but an increase in responding during the Same over Pre-CS $\left(F_{(1,13)}=41.55, p<\right.$ $0.001)$ and Different $\left(F_{(1,13)}=48.20, p<0.001\right)$.

Magazine entries during Pre-CS and CS periods were analyzed for group PIT and group CS (Fig. 1D). Both groups showed robust conditioned responding to the CS; animals increased magazine entries during the CS period compared with the Pre-CS period. A mixed ANOVA conducted using factors of Group (PIT and CS) and CS period (Pre-CS and CS) found no significant effect of Group $\left(F_{(1,25)}=0.50, p>0.05\right)$ but an effect of CS period $\left(F_{(1,25)}=54.24, p<0.001\right)$ and no significant interaction between Group and CS period $\left(F_{(1,25)}=3.38, p=0.08\right)$. Further analyses conducted in each group found a significant increase in magazine entries during the CS period in Group PIT $\left(F_{(1,13)}=\right.$ 15.67, $p<0.01)$ and Group CS $\left(F_{(1,12)}=41.38, p<0.001\right)$.

\section{Neuronal activity in the ventral pallidum}

Quantification of c-Fos-positive neurons in all animals in the hemisphere contralateral to the FG injection was performed in the VP-m and VP-l to assess the neuronal activity in each region for the three test conditions. Three sections for each region from each animal were quantified and Figure 2 shows images of c-Fos immunoreactivity in VP-m (top) and VP-l (bottom) for each test condition. The number of c-Fos-positive cells per square millimeter in VP-m and VP-l are presented in Figure 2, right. Oneway ANOVA conducted in the VP-m found a significant difference in c-Fos expression between groups $\left(F_{(2,37)}=4.29, p=\right.$ $0.02)$. Post hoc analyses found that, whereas there was no significant difference between groups LP and CS $\left(F_{(1,24)}=0.09, p=\right.$ $0.77)$, there was a significantly higher number of c-Fos-positive cells in group PIT compared with Group LP $\left(F_{(1,25)}=7.51, p=\right.$ $0.01)$ and Group CS $\left(F_{(1,25)}=5.42, p=0.03\right)$. In contrast, no significant effects emerged in the VP-l: comparable analysis conducted on the cell counts in that region found no significant difference in c-Fos expression between groups $\left(F_{(2,37)}=1.66, p=0.20\right)$.

$\mathrm{C}$-Fos expression in Group PIT was significantly correlated with net PIT performance in the VP-m $(r=0.60, p=0.02)$ but not in the VP-1 $(r=0.04, p=0.89$; Fig. $3 A)$. Conversely, whereas there was no significant correlation between $c$-Fos activation and overall lever pressing rates in animals in Groups PIT and LP in the VP-m $(r=0.32, p=0.10)$, there was a significant correlation in the VP-1 ( $r=0.46, p=0.02$; Fig. $3 B$ ) consistent with the VP-l's role in motor activity. No significant correlation between c-Fos expression and magazine entries during CS presentations was observed in the VP-m or VP-l for animals in the PIT and CS group consistent with the suggestion these circuits are not involved in Pavlovian conditioning per se.

\section{c-Fos activation of accumbal afferents to the medial ventral pallidum}

Neuronal activity in the NAc-S was quantified by itself and together with FG retrograde labeling from the VP-m. Only animals that had injections centered in the target area were included for analyses in the NAc-S (Group PIT; $n=13$, Group LP; $n=12$, Group CS; $n=12$ ). Figure $4 A$ shows a representative injection of FG in the VP-m and a schematic representation of the rostralcaudal spread of each injection is presented in Figure $4 B$. Dense retrogradely labeled neurons were observed in the NAc-S in the hemisphere ipsilateral to the injection. No contralateral labeling was visible. Figure $4 C$ demonstrates the coronal level at which sections in the NAc-S were selected for quantification and the dimension of confocal images taken for quantification. Examples of FG-labeled neurons, c-Fos-positive neurons, and colabeled neurons are shown on Figure $4 D$ with a magnification of the inset in the right panel. The mean number of FG-labeled neurons $(\mathrm{FG}+$ ), c-Fos-positive neurons (c-Fos + ), and percentage of c-Fos-positive neurons double-labeled with FG (\%c-Fos) per square millimeter for each group are represented in Figure $4 E$. The mean of total number of neurons counted over three sections, the average number of neurons per square millimeter and the percentage of double-labeled neurons for each group are also presented in Table 1. There were no significant differences in the number of FG-labeled neurons among the three conditions $\left(F_{(2,34)}=0.82, p=0.45\right)$. One-way ANOVA conducted on $c$-Fos + found an effect of Group $\left(F_{(2,34)}=5.61, p<0.01\right)$. Further simple effect analyses found no differences in c-Fos expression between groups LP and CS $\left(F_{(1,22)}<1\right)$ but significantly more c-Fos-positive cells in Group PIT than both Group LP $\left(F_{(1,23)}=7.52, p=0.01\right)$ and Group CS $\left(F_{(1,23)}=8.13, p=0.01\right)$. One-way ANOVA conducted on \%c-Fos also revealed an effect of Group $\left(F_{(2,34)}=23.44, p<0.001\right)$. Simple effect analyses found no differences in \%c-Fos between Groups LP and CS $\left(F_{(1,22)}=\right.$ $0.97, p=0.34$ ) but a significant increase in \%c-Fos in Group PIT 

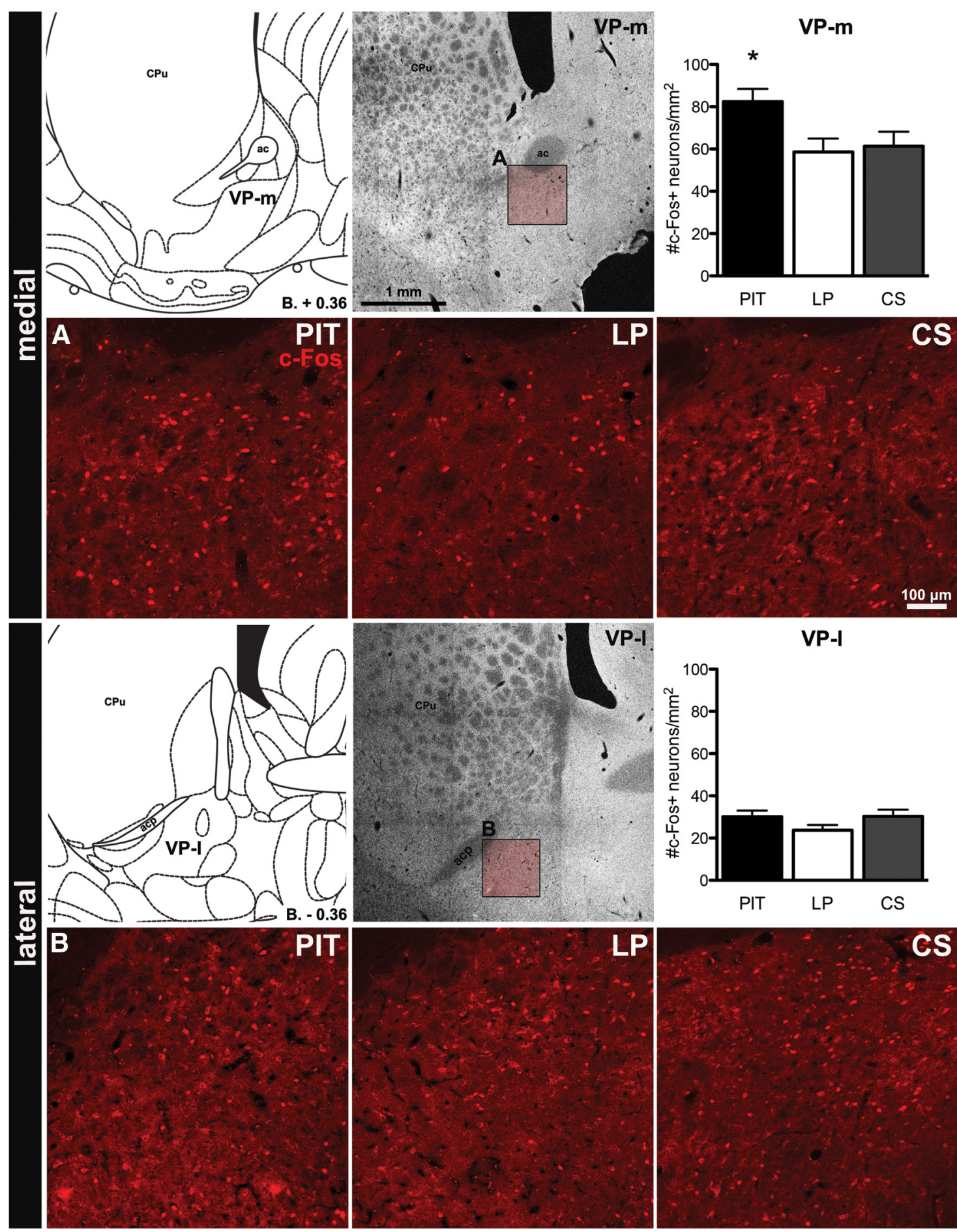

Figure 2. Experiment 1. c-Fos immunoreactivity in the VP-m (top) and VP-I (bottom). For each region, a schematic representation (Paxinos and Watson, 1998), a photomicrograph and quantification of c-Fos expression are displayed (top, left to right) with images of c-Fos immunoreactivity in each group (bottom, left to right). Insets in the photomicrograph show where images were taken for quantification in the VP-m (A) and VP-I (B). ac, Anterior commissure; acp, anterior commissure, posterior part; $\left(\mathrm{Pu}\right.$, caudate-putamen. Error bars indicate SEM. ${ }^{*} p<0.05$ compared with Groups LP and CS.

compared with Group LP $\left(F_{(2,23)}=24.99, p<0.001\right)$ and Group $\operatorname{CS}\left(F_{(2,23)}=48.33, p<0.001\right)$.

\section{Experiment 2: the effect of inactivation of the VP-m on PIT}

\section{Histology}

Figure $5 B$ shows a photomicrograph of a bilateral cannula placement in the VP-m and Figure $5 C$ shows a schematic of cannula placements within the VP-m of animals that were included in all analyses $(n=11)$. Three animals were excluded for wrong placement and two animals were excluded because of dislodgement of the guide cannulae between the two tests.

\section{Pavlovian and instrumental training}

All animals acquired both Pavlovian and instrumental conditioning. As described in Experiment 1, animals increased their 
conditioned responding during CS presentations during Pavlovian training and this effect increased over sessions. In the last session of Pavlovian training, the rate of magazine entries per minutes during the Pre-CS and CS period was $1.78 \pm 0.31$ and $11.38 \pm 0.93$, respectively. ANOVA revealed an effect of CS period $\left(F_{(1,10)}=\right.$ 209.33, $p<0.001)$, an effect of Session $\left(F_{(7,70)}=2.58, p<0.05\right)$, and a CS period by Session interaction $\left(F_{(7,70)}=12.84\right.$, $p<0.001$ ). Animals also increased their rates of responding on the levers as the ratio schedule increased in the first seven sessions (2 CRF, 3 RR5, and 2 RR10) with a lever press rate of $25.88 \pm 2.67$ per minute before they underwent surgery. Following recovery, animals were given three more RR10 sessions and on the final session they were pressing at a rate of $27.65 \pm$ 2.42. An ANOVA conducted on lever press rates during training revealed an effect of Session $\left(F_{(9,90)}=28.53, p<0.001\right)$. During instrumental retraining in between tests, responding returned to a similar rate to the last day of training and the rate of lever pressing was $30.35 \pm 2.07$ per minute.

\section{Pavlovian-instrumental transfer}

We assessed the effect of inactivating the VP-m in outcome specific PIT using a high $(0.5 \mathrm{mg} / \mathrm{ml})$ and low $(0.1 \mathrm{mg} / \mathrm{ml})$ concentration of the $\mathrm{GABA}_{\mathrm{A}}$ agonist muscimol. The results for the PIT test under high and low concentrations are presented in Figure $5 \mathrm{~A}$ as mean lever presses per minute during the Pre-CS, during the stimulus that predicted the same outcome as the response (Same) and during the stimulus that predicted the different outcome to the response (Different). Both doses yielded a similar pattern of results. When infused with vehicle, the rats showed a clear specific PIT effect and selectively increased responding on the lever that, during training, delivered the same outcome as that predicted by the CS. In contrast, when tested under either a high or low concentration of muscimol, the rats did not increase responding during the Same CS and responding remained at baseline levels throughout the test. When tested under a high concentration, ANOVA conducted on the high dose of Drug (separating the effects of vehicle or muscimol) and CS identity (separating the Pre-CS, Same CS, or Different CS periods) as factors revealed an effect of Drug $\left(F_{(1,10)}=26.30, p<0.001\right)$, an effect of CS identity $\left(F_{(2,20)}=19.29, p<0.001\right)$ and a Drug by CS identity interaction $\left(F_{(2,20)}=19.03, p<0.001\right)$. ANOVA conducted on the low dose of Drug and CS identity as factors revealed no effect of Drug $\left(F_{(1,10)}=1.70, p=0.22\right)$, but an effect of CS identity $\left(F_{(2,20)}\right.$ $=22.09, p<0.001)$ and a significant Drug by CS identity interaction $\left(F_{(2,20)}=7.57, p<0.01\right)$. Simple effect analyses conducted to further investigate these interactions found a significant increase in responding on the lever associated with the same outcome as the CS than the lever associated with the different outcome as predicted by the CS, when tested under both vehicle (VEH) $0.5\left(F_{(1,10)}=47.46\right.$, $p=0.002)$ and VEH $0.1\left(F_{(1,10)}=33.68, p=0.001\right)$ but no effect when tested under muscimol (MUS) $0.5 \mathrm{mg} / \mathrm{ml}\left(F_{(1,10)}=0.59\right.$, $p=0.46)$ or MUS $0.1 \mathrm{mg} / \mathrm{ml}\left(F_{(1,10)}=1.47, p=0.25\right)$.

\section{Experiment 3: effect of disconnection of the NAc-S and VP-m on PIT}

\section{Histology}

Figure $6 C$ shows a photomicrograph of the angled cannula placement in the NAc-S and VP-m and Figure $6 D$ shows a schematic illustration of the cannulae placements within the NAc-S and VP-m for all of the rats included in the analyses of Experiment 3. Seven rats were excluded from further analyses for inaccurate placement of one or other cannula. Group sizes following exclusions were as follows: Group Contralateral $(n=8)$ and Group Ipsilateral $(n=11)$. Assessment of the spread of the fluorescence signal induced by the muscimol infusion found that the fluorescence remained localized to the region of infusion and did not spread to adjacent structures and was segregated in the A-P plane between the NAc-S and the VP-m. Reconstruction of a representative infusion is shown in Figure $6 B$ which shows a coronal view of contralateral injections in the NAc-S and VP-m at different anterior to posterior levels; from bregma +1.60 to +0.20 . It is clear from this figure that the spread of the drug did not overlap into neighboring structures for either infusion.

\section{Pavlovian and instrumental training}

Animals were counterbalanced into either ipsilateral or contralateral cannulae groups based on their training performance and therefore had no differences in their Pavlovian and instrumental training. During Pavlovian conditioning animals showed an increasing conditioned response to the CS over sessions. A mixed ANOVA with Group, CS period, and Session as factors 

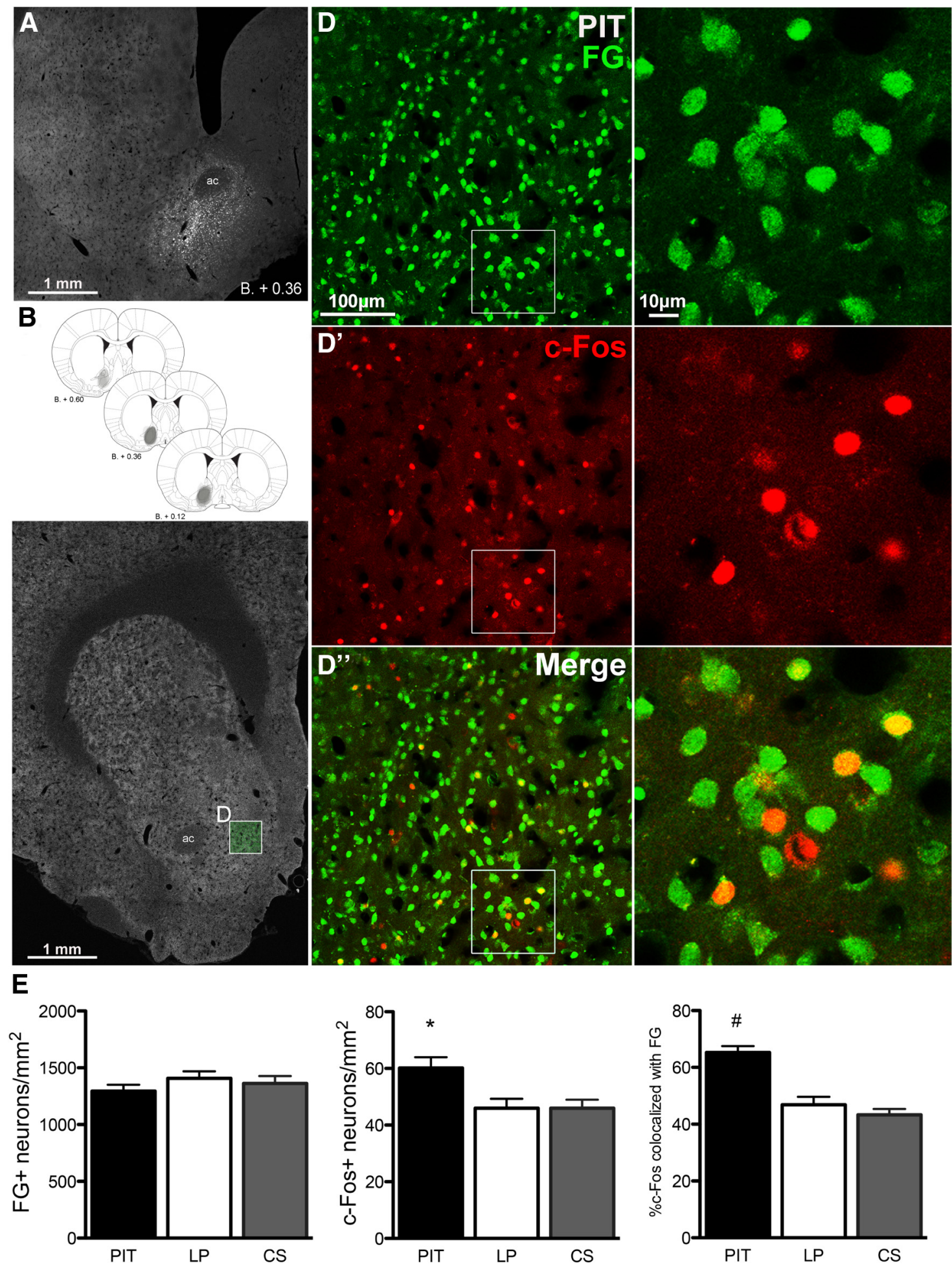

Figure 4. Experiment 1.FG injection in the VP-m and retrograde labeling in the NAc-S. A, Photomicrograph of a representative FG injection site in the VP-m. B, Schematic representation (Paxinos and Watson, 2007) of the rostral-caudal spread of the FG injection for all animals. C, Photomicrograph of a section processed for quantification in NAc-S ( $\boldsymbol{D}$, inset). $\boldsymbol{D}$, Right, FG retrograde labeling, c-Fos immunoreactivity $\left(\boldsymbol{D}^{\prime}\right)$, and double-labeling $\left(\boldsymbol{D}^{\prime \prime}\right)$ in the NAc-S; left shows magnifications of insets. $\boldsymbol{E}$, Number of FG-positive neurons (FG + ), c-Fos-positive neurons (c-Fos + ), and the percentage of c-Fos-positive neurons colocalized with FG in the NAc-S for each group. Error bars indicate SEM. ${ }^{*} p<0.05$ compared with Groups LP and CS. \#p $<0.01$ compared with Groups LP and CS.

revealed an effect of CS period $\left(F_{(1,17)}=174.28, p<0.001\right)$, an effect of Session $\left(F_{(7,119)}=51.34, p<0.001\right)$, and a CS period by Session interaction $\left(F_{(7,119)}=67.54, p<0.001\right)$. There was no significant effect of Group or any interactions involving Group as a factor (all $F$ values $<1$ ). On the last training session, the two groups were entering the magazine at similar rates during the Pre-CS and CS periods; Group Ipsilateral: $2.33 \pm 0.49$ and $21.90 \pm 1.93$ per minute, and Group Contralateral: $2.41 \pm 0.55$ and $21.19 \pm 3.03$ per minute, respectively. During instrumental training, animals increased their rates of responding as the ratio 
Table 1. Experiment 1. The number of c-Fos positive neurons in the NAc-S during PIT and the proportion of c-Fos positive neurons retrogradely labeled with Fluoro-gold infused into the VPm

\begin{tabular}{|c|c|c|c|c|c|c|c|}
\hline \multirow[b]{2}{*}{ Group } & \multicolumn{3}{|c|}{ Total number of neurons ${ }^{a}$} & \multicolumn{3}{|l|}{ Average per $\mathrm{mm}^{2}$} & \multirow{2}{*}{$\begin{array}{l}\text { \%double-labelled } \\
\text { cFos }+ \text { FG }+\end{array}$} \\
\hline & $\mathrm{FG}+$ & cFos + & cFos +FG + & $\mathrm{FG}+$ & cFos + & cFos + FG + & \\
\hline PIT & 699.57 (28.61) & $32.29(1.94)$ & $21.29(1.60)$ & $1290.41(52.78)$ & $59.55(3.57)$ & $39.26(2.95)$ & 65.65 (2.18) \\
\hline LP & 762.92 (33.72) & $24.92(1.81)$ & $11.58(1.03)$ & $1407.26(62.19)$ & $45.96(3.34)$ & $21.37(1.91)$ & $46.83(2.78)$ \\
\hline CS & $738.33(35.75)$ & $24.92(1.64)$ & $10.75(0.88)$ & $1361.91(65.95)$ & $45.96(3.02)$ & $19.83(1.62)$ & $43.30(2.04)$ \\
\hline
\end{tabular}

${ }^{a}$ Group means of the sum of three sections. Values represent the mean ( \pm 1 SEM).

\section{A}
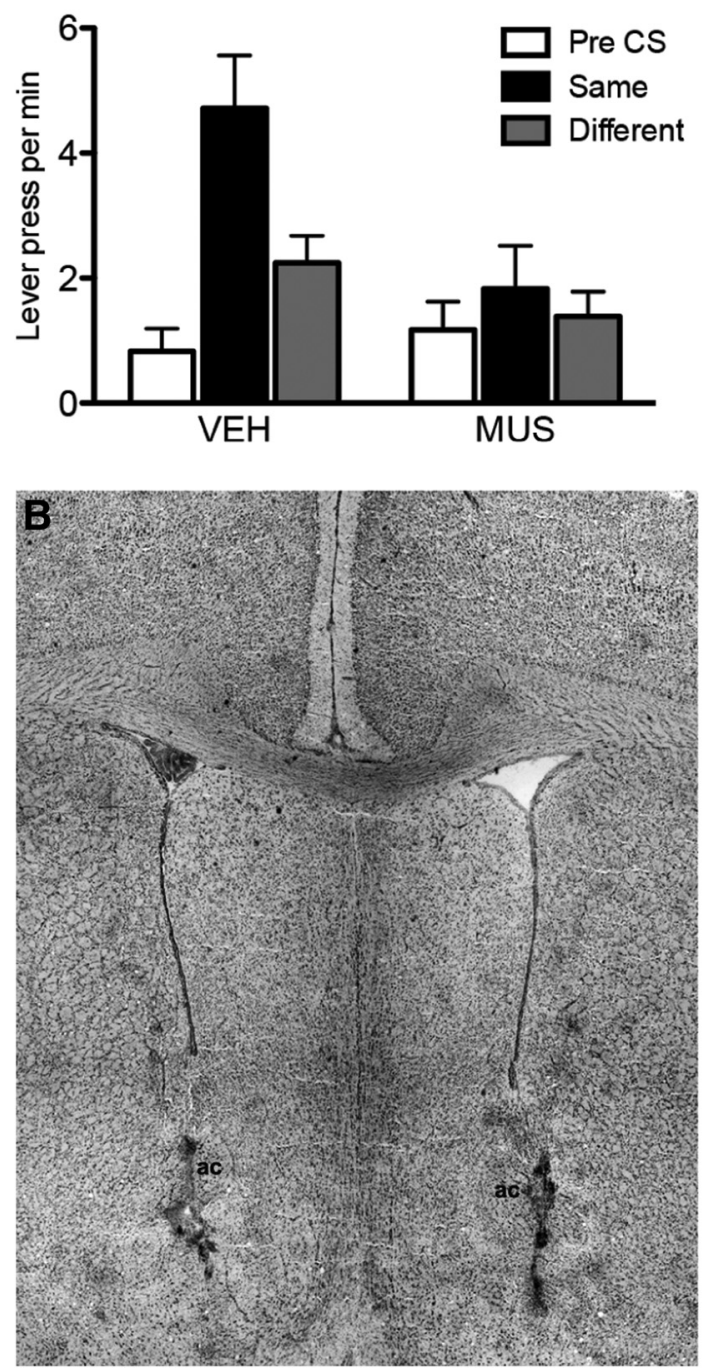

Muscimol $0.5 \mathrm{mg} / \mathrm{ml}$

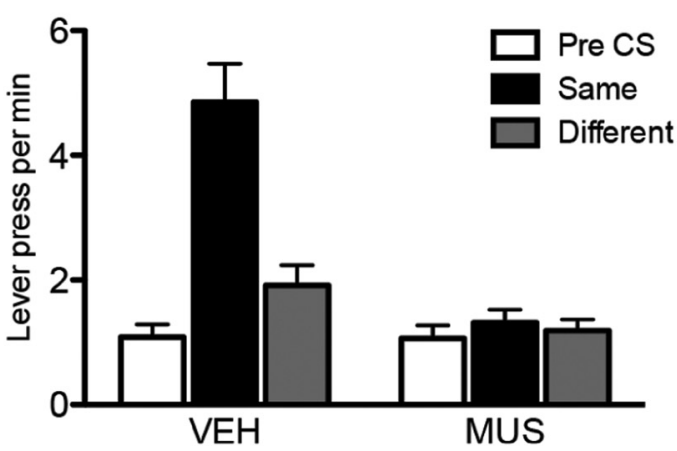

C

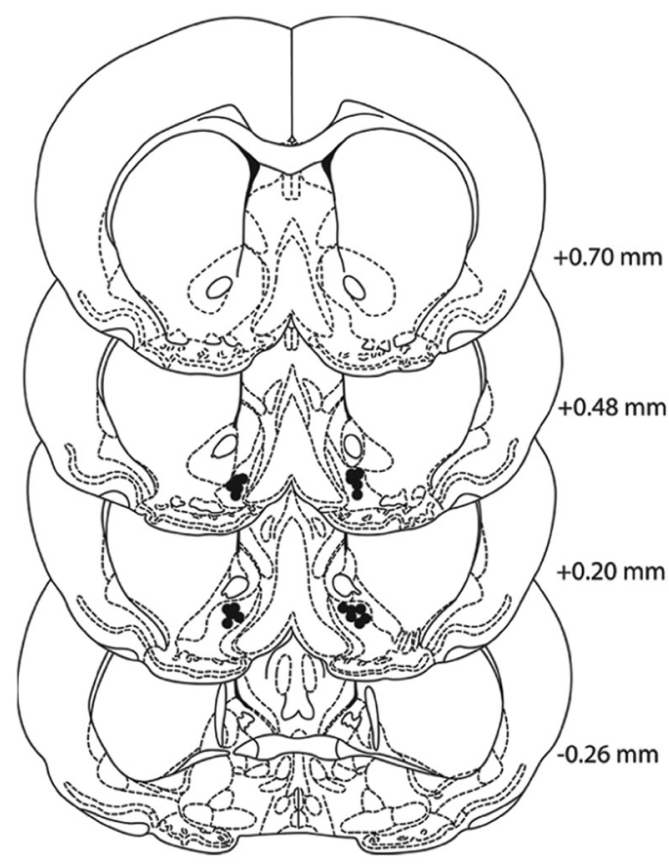

Figure 5. Experiment 2. Inactivation of the VP-m during PIT. $A$, Effect of a low (left) and high (right) dose of MUS infused into VP-m on PIT showing lever presses per minute when rats were given an infusion of VEH or MUS. Performance is shown as lever pressing per minute during the Pre-CS period (Pre-CS), and separately during the CS period for responding on the lever that, in training, delivered the same outcome as that predicted by the $\mathrm{CS}$ (Same) and on the lever that had delivered the different outcome (Different). $\boldsymbol{B}$, Photomicrograph of a representative cannula placement in the VP-m. C, Placements of injection cannula tips in the VP-m (black dots) for all animals in Experiment 2. Error bars indicate SEM.

schedule increased over days. Despite receiving guide cannulae implants contralaterally or ipsilaterally during instrumental training, there were no differences in responding between the two groups. A mixed ANOVA of Group and Session revealed an effect of Session $\left(F_{(9,153)}=60.65, p<0.001\right)$ but no effect of Group $\left(F_{(1,17)}=0.13, p=0.73\right)$ and no interaction between these factors $\left(F_{(9,153)}=0.55, p=0.84\right)$. In the last session of instrumental training following surgery, both ipsilateral and contralateral groups were responding on the levers at similar rates of $29.86 \pm$ 3.00 and $27.52 \pm 2.59$ per minute, respectively.

\section{Pavlovian-instrumental transfer}

We assessed the effect of NAc-S to VP-m disconnection on specific PIT by inactivating both structures either in the ipsilateral hemisphere or in contralateral hemispheres before test. The results of the ipsilateral and contralateral inactivation are presented in Figure 6A plotted as lever presses per min during: the Pre-CS period, the CS predicting the same outcome as the lever (Same), and the CS predicting the different outcome from the lever (Different). It is clear from the figure that when animals received ipsilateral infusions of either VEH or fMUS, they showed a strong 
A

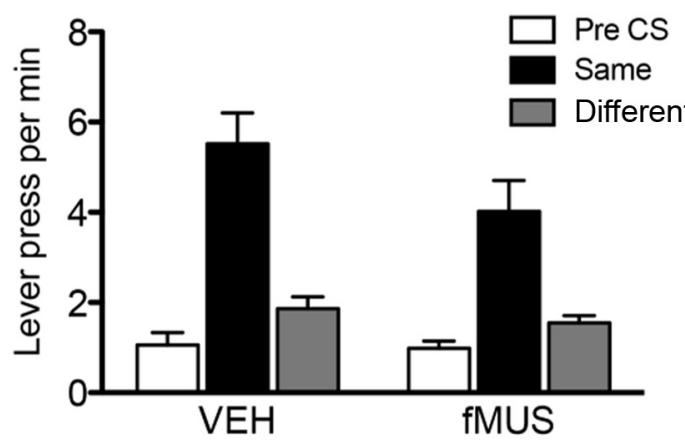

Contralateral

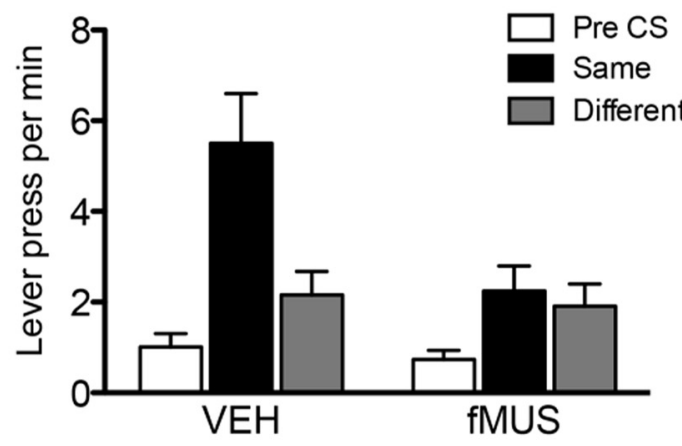

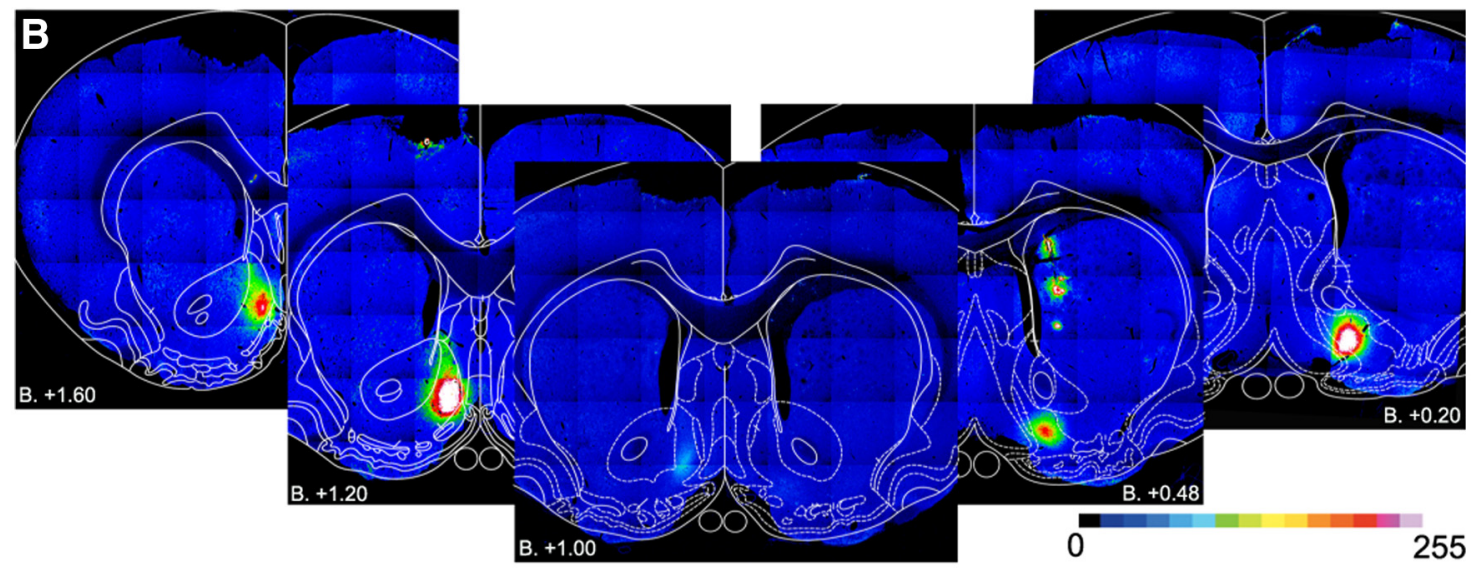
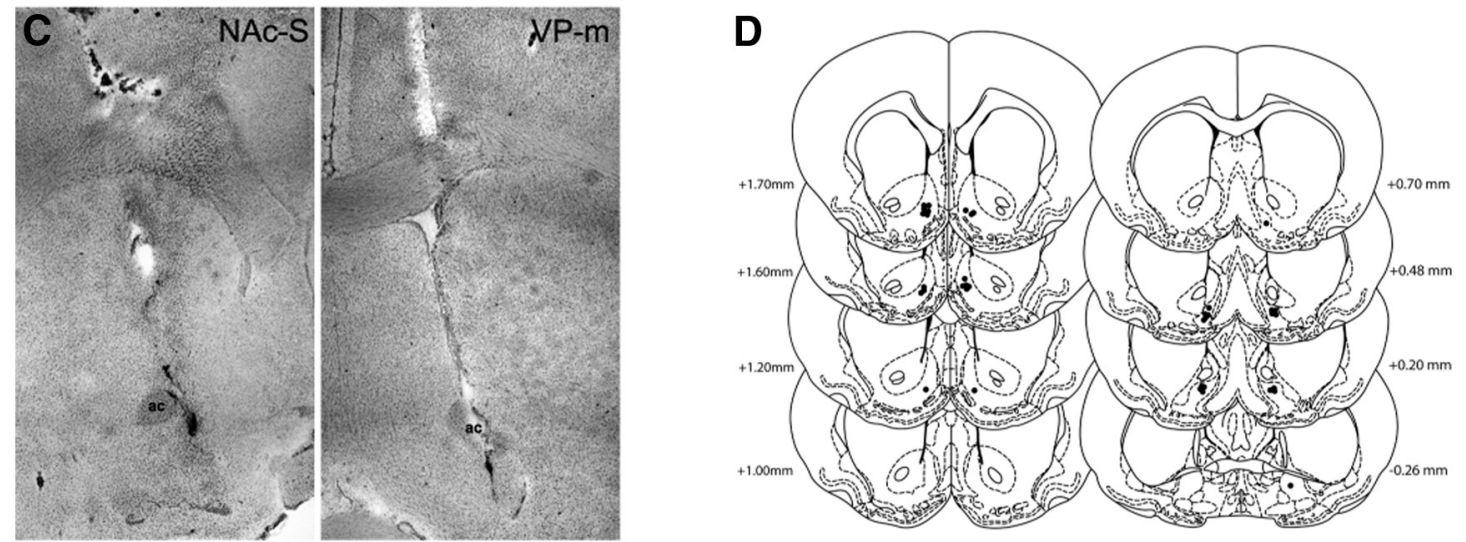

Figure 6. Experiment 3. Disconnection of Nac-S and VP-m during PIT.A, Effect of fMUS infused ipsilaterally (left) and contralaterally (right) on PIT. Performance is shown as lever presses per minute during the Pre-CS period (Pre-CS) and separately during the CS period, for responding on the lever that, in training, delivered the same outcome as that predicted by the CS (Same) and on the lever that had delivered the different outcome (Different). B, Visualization of the diffusion of fMUS in the NAc-S (left to center) and VP-m (right to center) with a greyscale value of $0-255$. Note that the infusion volumes and coordinates ensured that these did not overlap in the A-P dimension. $\boldsymbol{C}$, Photomicrograph of a representative angled cannulae placement in NAc-S and VP-m. $\boldsymbol{D}$, Placements of injection cannula tips (black dots) in the NAc-S (left) and the VP-m (right) for all rats in Experiment 3. Error bars indicate SEM.

specific PIT effect. A different pattern of result emerged in the contralateral condition: where, rats given $\mathrm{VEH}$ infusions in contralateral hemispheres showed a clear specific PIT effect, this effect was abolished when the rats were infused with fMUS in the NAc-S and VP-m contralaterally. ANOVA conducted on the ipsilateral group using factors of Drug (separating the effects of vehicle and muscimol) and of CS identity (separating Pre-CS, same CS and different CS periods) revealed no effect of Drug $\left(F_{(1,10)}=\right.$ $1.61, p=0.24)$, an effect of CS identity $\left(F_{(2,20)}=81.83, p<0.001\right)$ and no significant interaction between these factors $\left(F_{(2,20)}=1.36\right.$, $p=0.28$ ). Simple effects analyses conducted on responding revealed a significant increase in responding on the lever that, in training, delivered the outcome predicted by that $\mathrm{CS}$ when rats received infusions into the NAc-S and VP-m ipsilaterally with VEH $\left(F_{(1,10)}=\right.$ $34.74, p<0.001)$ or with fMUS $\left(F_{(1,10)}=33.60, p=0.008\right)$. In contrast, comparable ANOVA conducted on the group given the infusions in contralateral hemispheres revealed a very different effect: disconnection of NAc-S and VP-m in Group Contralateral revealed no effect of Drug $\left(F_{(1,7)}=2.38, p=0.17\right)$, an effect of CS identity $\left(F_{(2,14)}=23.01, p<0.001\right)$ and a significant Drug by CS identity interaction $\left(F_{(2,14)}=5.39, p=0.02\right)$. Simple effects analyses found a significant increase in responding on the lever associated with the same outcome as the CS when rats received contralateral infusions of $\operatorname{VEH}\left(F_{(1,7)}=22.16, p<0.002\right)$, whereas there was no 
such increase in responding when they received a contralateral infusion of fMUS $\left(F_{(1,7)}=0.21, p=0.66\right)$.

\section{Discussion}

The current experiments suggest that the VP-m, through its connections with the NAc-S, mediates outcome-specific Pavlovianinstrumental transfer. First, we identified a region in the rostromedial VP in which c-Fos activity was both elevated during the test of specific PIT, relative to lever press and CS exposed controls, and correlated with size of the PIT effect. In contrast, although c-Fos activity in the VP-l correlated with the rate of lever pressing during the PIT test, it was unrelated to the PIT effect and c-Fos expression in a group exposed to PIT was similar to that in groups exposed to either the CS alone or to the levers alone. Furthermore, we found clear evidence that the NAc-S to VP-m pathway was activated during the PIT test. The infusion of FG into the VP-m produced retrograde labeling of neurons in the NAc-S, and after exposure to the test, we found a significantly higher number of FG and c-Fos double-labeled neurons in the NAc-S in a group tested for PIT than in groups exposed to the levers or the CSs alone during the test.

A regional change in c-Fos activity, however, does not confirm the region mediates the specific function associated with that change. To confirm the VP-m was involved in PIT, therefore, we inactivated it using bilateral infusions of muscimol before the PIT test and found that these infusions rendered the rats unable to exhibit specific PIT. Second, to confirm the importance of the NAc-S to VP-m pathway, we disconnected it during the PIT test by infusing fluorescence tagged muscimol into the NAc-S and VP-m in contralateral hemispheres and compared this treatment with a group given the infusions ipsilaterally as a control. When rats received the infusions into the two regions ipsilaterally, leaving communication between these structures intact in the contralateral hemisphere, the rats were able to perform normally and showed specific PIT. In contrast, disconnection of the NAc-S to VP-m pathway by inactivating these structures contralaterally abolished PIT. This pattern of results confirms the functional importance of the NAc-S to VP-m pathway and suggests that the effects of bilateral VP-m inactivation were not due to a general motor impairment; animals that received infusions of muscimol into the NAc-S and VP-m ipsilaterally were able to show specific PIT, whereas those with similar infusions but made contralaterally could not.

\section{The ventral pallidum and reward}

To date, there has been no systematic assessment of the functions of specific subregions of the VP. Although anatomical studies have often separated the structure into different regions based on their efferent and afferent connections and immunohistochemistry (Zahm and Heimer, 1990; Groenewegen et al., 1993; Zahm et al., 1996; Usuda et al., 1998), it has proven difficult to manipulate regionally using traditional approaches. The majority of research investigating the function of the VP has examined the structure as a whole and, in similar fashion to the nucleus accumbens, has generally concluded it mediates the effect of incentive processes on performance as part of the limbic-motor interface. For example, lesions to the VP produce deficits in conditioning a place preference for sucrose (McAlonan et al., 1993) and in the reversal of stimulus-outcome associations in an odor discrimination task (Ferry et al., 2000). In addition, the VP has been argued to be important for the reinstatement of instrumental responding after extinction (McFarland and Kalivas, 2001; Mahler and Aston-Jones, 2012) and disrupting GABA transmission by phar- macologically manipulating the VP differentially affects feeding and effort-based choice. (Stratford et al., 1999; Farrar et al., 2008). Furthermore, populations of neurons in the VP respond to both reward and cues that predict reward (Tindell et al., 2004), whereas in primates, neurons in the VP have been found to encode the expected value of the reward a CS predicts (Tachibana and Hikosaka, 2012).

Of the few studies that have investigated regional differences within the VP, opioid manipulations have been found to increase the hedonic impact of tastes in the caudolateral VP but have the opposite effect in the rostromedial VP (Smith and Berridge, 2005). Likewise, microinjections of neurotensin into the VP, which is colocalized with the NAc-S projection to the VP-m, have been shown to selectively inhibit cue-induced reinstatement but not drug-induced reinstatement (Torregrossa and Kalivas, 2008). More recently, there have been reports of differences in the electrophysiological properties of neurons in the two regions during cocaine self stimulation as well as in the morphological characteristics of neurons in the rostromedial VP (Kupchik and Kalivas, 2012; Root et al., 2013). The specific PIT procedure used in the current experiments was intended to isolate the Pavlovian incentive motivational properties of a stimulus that has been associated with a specific reward from those that influence goal directed actions. As such, the results from the current study demonstrate an additional dichotomy in the VP; that c-Fos expression in the medial, rather than the lateral VP is associated with PIT and this neuronal activity in the VP-m critically mediates the effect of Pavlovian cues on choice.

\section{The ventral striato-pallidal pathway}

Functionally, parallel circuits from NAc-S and NAc-C have been shown previously to mediate different aspects of the way Pavlovian conditioning influences instrumental actions with the NAc-S mediating specific PIT and the NAc-C general PIT, a form of PIT associated with general motivational arousal as opposed to the highly selective predictions of specific outcomes (Hall et al., 2001; Corbit and Balleine, 2011). Both regions of the accumbens also receive inputs from the basolateral amygdala (BLA) that affect instrumental action and yet it is the BLA to NAc-S pathway that controls specific PIT (Shiflett and Balleine, 2010). From a functional perspective, therefore, is not surprising that signals from distinct regions of nucleus accumbens, project to separate regions in the VP and that, in the current study, we found that a significant proportion of c-Fos-positive neurons in the NAc-S projected to the VP-m. Indeed, a single axon tracing study of NAc-S neurons found that all short- and long-range neurons traced from the NAc-S targeted the VP-m, with a number of extending collaterals further afield, whereas a substantial number of shortranged neurons terminated in the VP-m (Tripathi et al., 2012). Unfortunately, the large bulk tracer injections used in the current study were not able to differentiate whether activated neurons in the NAc-S terminated in the VP-m or continued to other structures, as both short-ranged and long-ranged neurons were comingled. However, in addition to the fact that long-range targets of the NAc-S such as the ventral tegmental area (VTA) or lateral hypothalamus are also targets of the VP itself, results from our double-labeling study, together with the reduction in specific PIT when disconnected from the NAc-S, support the suggestion that the VP-m is a critical structure in a circuit mediating specific PIT.

The current experiments used a choice test to examine the role of the NAc-S and VP-m pathway in specific PIT and, as such, choices could reflect a bias toward the lever delivering the pre- 
dicted outcome or away from the lever predicting the different outcome. Given the fact that the NAc-S projection to the VP-m is GABAergic, it may be thought that this pathway is more involved in the latter than the former. However, excitatory activation of both regions may be possible through several mechanisms. For example, NAc-S control of the VP-m could emerge through its regulation of other inputs to the VP-m; in addition to the GABAergic input from the NAc-S, glutamatergic and dopaminergic inputs from a variety of structures have been reported (Maslowski and Napier, 1991; Klitenick et al., 1992; Zaborszky et al., 1997) and Kupchik and Kalivas (2012) have recently described a subtype of GABAergic neuron in the rostromedial VP regulated by a glutamatergic input. Alternatively, the NAc-S could result in excitatory VP-m activity through the interaction of multiple GABAergic synapses involving GABAergic interneurons or converging inhibitory collaterals. It is interesting to note, for example, that both D1- and D2-expressing medium spiny neurons in the NAc-S project to the VP-m ( $\mathrm{Lu}$ et al., 1998), allowing dopamine and a number of coexpressed neuropeptides in the NAc-S, including neurotensin, substance $\mathrm{P}$ and various opioids, to modulate the release of GABA to alter the activity of the VP-m and its downstream targets (Chrobak and Napier, 1993; Napier et al., 1995; Olive et al., 1997; Napier and Mitrovic, 1999; Torregrossa and Kalivas, 2008). With regard to the latter, there are distinct projections from the VP-m to the dopamine neurons in the VTA (Hjelmstad et al., 2013) but also to mediodorsal thalamus (Young et al., 1984; Zahm, 2000; Tripathi et al., 2012), the latter providing a unique side loop for information from the NAc-S to reach the cortex via the VP-m instead of the midbrain. Interestingly, both the VTA and mediodorsal thalamus have been implicated in specific PIT; albeit in slightly different ways (Corbit et al., 2007; Ostlund and Balleine, 2008) and the organization of these connections provides a promising target for future studies.

\section{References}

Austin MC, Kalivas PW (1990) Enkephalinergic and GABAergic modulation of motor activity in the ventral pallidum. J Pharmacol Exp Ther 252:1370-1377. Medline

Chrobak JJ, Napier TC (1993) Opioid and GABA modulation of accumbens-evoked ventral pallidal activity. J Neural Transm Gen Sect 93:123-143. CrossRef Medline

Corbit LH, Muir JL, Balleine BW (2001) The role of the nucleus accumbens in instrumental conditioning: evidence of a functional dissociation between accumbens core and shell. J Neurosci 21: 3251-3260. Medline

Corbit LH, Balleine BW (2005) Double dissociation of basolateral and central amygdala lesions on the general and outcome-specific forms of Pavlovian-instrumental transfer. J Neurosci 25:962-970. CrossRef Medline

Corbit LH, Janak PH (2007) Inactivation of the lateral but not medial dorsal striatum eliminates the excitatory impact of Pavlovian stimuli on instrumental responding. J Neurosci 27:13977-13981. CrossRef Medline

Corbit LH, Janak PH, Balleine BW (2007) General and outcome-specific forms of Pavlovian-instrumental transfer: the effect of shifts in motivational state and inactivation of the ventral tegmental area. Eur J Neurosci 26:3141-3149. CrossRef Medline

Corbit LH, Balleine BW (2011) The general and outcome-specific forms of Pavlovian-instrumental transfer are differentially mediated by the nucleus accumbens core and shell. J Neurosci 31:11786-11794. CrossRef Medline

Farrar AM, Font L, Pereira M, Mingote S, Bunce JG, Chrobak JJ, Salamone JD (2008) Forebrain circuitry involved in effort-related choice: injections of the GABAA agonist muscimol into ventral pallidum alter response allocation in food-seeking behavior. Neuroscience 152:321-330. CrossRef Medline

Ferry AT, Lu XC, Price JL (2000) Effects of excitotoxic lesions in the ventral striatopallidal-thalamocortical pathway on odor reversal learning: inabil- ity to extinguish an incorrect response. Exp Brain Res 131:320-335. CrossRef Medline

Groenewegen HJ, Berendse HW, Haber SN (1993) Organization of the output of the ventral striatopallidal system in the rat: ventral pallidal efferents. Neuroscience 57:113-142. CrossRef Medline

Hall J, Parkinson JA, Connor TM, Dickinson A, Everitt BJ (2001) Involvement of the central nucleus of the amygdala and nucleus accumbens core in mediating Pavlovian influences on instrumental behaviour. Eur J Neurosci 13:1984-1992. CrossRef Medline

Hjelmstad GO, Xia Y, Margolis EB, Fields HL (2013) Opioid modulation of ventral pallidal afferents to ventral tegmental area neurons. J Neurosci 33:6454-6459. CrossRef Medline

Kalivas PW, Churchill L, Romanides A (1999) Involvement of the pallidalthalamocortical circuit in adaptive behavior. Ann N Y Acad Sci 877:6470. CrossRef Medline

Klitenick MA, Deutch AY, Churchill L, Kalivas PW (1992) Topography and functional role of dopaminergic projections from the ventral mesencephalic tegmentum to the ventral pallidum. Neuroscience 50:371-386. CrossRef Medline

Kupchik YM, Kalivas PW (2012) The rostral subcommissural ventral pallidum is a mix of ventral pallidal neurons and neurons from adjacent areas: an electrophysiological study. Brain Struct Funct. Advance online publication. doi:10.1007/s00429-012-0471-9. CrossRef Medline

Laurent V, Leung B, Maidment N, Balleine BW (2012) Mu- and deltaopioid-related processes in the accumbens core and shell differentially mediate the influence of reward-guided and stimulus-guided decisions on choice. J Neurosci 32:1875-1883. CrossRef Medline

Lu XY, Ghasemzadeh MB, Kalivas PW (1998) Expression of D1 receptor, $D 2$ receptor, substance $P$ and enkephalin messenger RNAs in the neurons projecting from the nucleus accumbens. Neuroscience 82:767-780. Medline

Mahler SV, Aston-Jones GS (2012) Fos activation of selective afferents to ventral tegmental area during cue-induced reinstatement of cocaine seeking in rats. J Neurosci 32:13309-13326. CrossRef Medline

Maslowski RJ, Napier TC (1991) Dopamine D1 and D2 receptor agonists induce opposite changes in the firing rate of ventral pallidal neurons. Eur J Pharmacol 200:103-112. CrossRef Medline

McAlonan GM, Robbins TW, Everitt BJ (1993) Effects of medial dorsal thalamic and ventral pallidal lesions on the acquisition of a conditioned place preference: further evidence for the involvement of the ventral striatopallidal system in reward-related processes. Neuroscience 52:605-620. CrossRef

McFarland K, Kalivas PW (2001) The circuitry mediating cocaine-induced reinstatement of drug-seeking behavior. J Neurosci 21:8655-8663.

Napier TC, Mitrovic I (1999) Opioid modulation of ventral pallidal inputs. Ann N Y Acad Sci 877:176-201. CrossRef Medline

Napier TC, Mitrovic I, Churchill L, Klitenick MA, Lu XY, Kalivas PW (1995) Substance $\mathrm{P}$ in the ventral pallidum: projection from the ventral striatum, and electrophysiological and behavioral consequences of pallidal substance p. Neuroscience 69:59-70. CrossRef Medline

Olive MF, Anton B, Micevych P, Evans CJ, Maidment NT (1997) Presynaptic versus postsynaptic localization of $\mathrm{mu}$ and delta opioid receptors in dorsal and ventral striatopallidal pathways. J Neurosci 17:7471-7749. Medline

Ostlund SB, Balleine BW (2007) The contribution of orbitofrontal cortex to action selection. Ann N Y Acad Sci 1121:174-192. CrossRef Medline

Ostlund SB, Balleine BW (2008) Differential involvement of the basolateral amygdala and mediodorsal thalamus in instrumental action selection. J Neurosci 28:4398-4405. CrossRef Medline

Paxinos G, Watson C (1998) The rat brain in stereotaxic coordinates, Ed 4. New York: Academic

Paxinos G, Watson C (2007) The rat brain in stereotaxic coordinates, Ed 6. Amsterdam: Academic.

Root DH, Ma S, Barker DJ, Megehee L, Striano BM, Ralston CM, Fabbricatore AT, West MO (2013) Differential roles of ventral pallidum subregions during cocaine self-administration behaviors. J Comp Neurol 521: 558-588. CrossRef Medline

Shiflett MW, Balleine BW (2010) At the limbic-motor interface: disconnection of basolateral amygdala from nucleus accumbens core and shell reveals dissociable components of incentive motivation. Eur J Neurosci 32:1735-1743. CrossRef Medline

Smith KS, Berridge KC (2005) The ventral pallidum and hedonic reward: 
neurochemical maps of sucrose "liking" and food intake. J Neurosci 25: 8637-8649. CrossRef Medline

Smith KS, Tindell AJ, Aldridge JW, Berridge KC (2009) Ventral pallidum roles in reward and motivation. Behav Brain Res 196:155-167. CrossRef Medline

Stratford TR, Kelley AE, Simansky KJ (1999) Blockade of GABA A receptors in the medial ventral pallidum elicits feeding in satiated rats. Brain Res 825:199-203. CrossRef Medline

Tachibana Y, Hikosaka O (2012) The primate ventral pallidum encodes expected reward value and regulates motor action. Neuron 76:826-837. CrossRef Medline

Tindell AJ, Berridge KC, Aldridge JW (2004) Ventral pallidal representation of Pavlovian cues and reward: population and rate codes. J Neurosci 24:1058-1069. CrossRef Medline

Torregrossa MM, Kalivas PW (2008) Neurotensin in the ventral pallidum increases extracellular GABA and differentially affects cue- and cocaineprimed reinstatement. J Pharmacol Exp Ther 325:556-566. CrossRef Medline

Tripathi A, Prensa L, Cebrián C, Mengual E (2010) Axonal branching patterns of nucleus accumbens neurons in the rat. J Comp Neurol 518:46494673. CrossRef Medline

Tripathi A, Prensa L, Mengual E (2012) Axonal branching patterns of ventral pallidal neurons in the rat. Brain Struct Funct. Advance online publication. doi:10.1007/s00429-012-0451-0. CrossRef Medline
Usuda I, Tanaka K, Chiba T (1998) Efferent projections of the nucleus accumbens in the rat with special reference to subdivision of the nucleus: biotinylated dextran amine study. Brain Res 797:73-93. CrossRef Medline

Young WS 3rd, Alheid GF, Heimer L (1984) The ventral pallidal projection to the mediodorsal thalamus: a study with fluorescent retrograde tracers and immunohistofluorescence. J Neurosci 4:1626-1638. Medline

Zaborszky L, Gaykema RP, Swanson DJ, Cullinan WE (1997) Cortical input to the basal forebrain. Neuroscience 79:1051-1078. CrossRef Medline

Zahm DS (2000) An integrative neuroanatomical perspective on some subcortical substrates of adaptive responding with emphasis on the nucleus accumbens. Neurosci Biobehav Rev 24:85-105. Medline

Zahm DS, Heimer L (1990) Two transpallidal pathways originating in the rat nucleus accumbens. J Comp Neurol 302:437-446. Medline

Zahm DS, Grosu S, Irving JC, Williams EA (2003) Discrimination of striatopallidum and extended amygdala in the rat: a role for parvalbumin immunoreactive neurons? Brain Res 978:141-154. CrossRef Medline

Zahm DS, Williams E, Wohltmann C (1996) Ventral striatopallidothalamic projection: IV. Relative involvements of neurochemically distinct subterritories in the ventral pallidum and adjacent parts of the rostroventral forebrain. J Comp Neurol 364:340 -362. CrossRef Medline

Zhou L, Furuta T, Kaneko T (2003) Chemical organization of projection neurons in the rat accumbens nucleus and olfactory tubercle. Neuroscience 120:783-798. CrossRef Medline 